\title{
Seleção de um modelo de referência de PDP para uma empresa de autopeças através de um método de auxílio à decisão por múltiplos critérios
}

\author{
Jefferson Olegário de Paula ${ }^{\mathrm{a} *}$, Carlos Henrique Pereira Mello ${ }^{\mathrm{b}}$ \\ a*jeffersonolegario@uol.com.br, UNIFEl, Brasil \\ bcarlos.mello@unifei.edu.br, UNIFEl, Brasil
}

\begin{abstract}
Resumo
0 processo de desenvolvimento de produtos (PDP) é um fator crítico de sucesso no lançamento de um novo produto para atender os requisitos dos clientes. A adoção de um modelo de referência serve como um guia detalhado para auxiliar as organizações na gestão e sistematização do PDP, através da sequência padronizada de etapas que são adaptadas de acordo com o tipo de produto que a empresa desenvolve. 0 objetivo deste artigo é selecionar um modelo de referência do processo de desenvolvimento de produtos para uma empresa do ramo de autopeças através de um método de auxílio à decisão por multicritério denominado Analytic Hierarchy Process (AHP). 0 método de pesquisa utilizado foi a modelagem, que diante de seis alternativas, três critérios e julgamento de cinco especialistas resultou na seleção do modelo de Rozenfeld et al. (2006) como o modelo mais indicado para realizar a análise do PDP da empresa objeto de estudo em pesquisas futuras.
\end{abstract}

Palavras-chave

Gestão do processo de desenvolvimento de produtos. Modelos de referência. AHP.

\section{Introdução}

Para aumentar os níveis de competitividade, pequenas, médias e grandes empresas investem profundamente no desenvolvimento de seus produtos, tanto na criação de produtos inovadores como no aperfeiçoamento dos já existentes. Diante de uma massa de clientes cada vez mais exigente, com necessidades, gostos e escolhas diferentes, existe a crescente demanda por novos produtos, de alta qualidade e com tempo e custos de desenvolvimento reduzidos, como parte integrante dos objetivos estratégicos das empresas. A adoção de um processo sistemático de desenvolvimento de produtos (PDP) é considerada atualmente como um fator crítico de sucesso no alcance desses objetivos estratégicos. Um dos segmentos de mercado que atua fortemente com o PDP é a indústria de autopeças, até mesmo pelas constantes reestilizações nos veículos promovidas pela indústria automobilística.
Os avanços tecnológicos nos veículos incluem o desenvolvimento de muitas características como desempenho, conforto, segurança, preservação ambiental e redução de custos. Esses aspectos, segundo Seidel, Loch e Chahil (2005), tendem a aumentar a complexidade dos produtos, levando as montadoras e seus fornecedores de autopeças a adotarem sistemas de modularidade, devido à explosão de variantes no mercado e do grande número de novas tecnologias de componentes que surgem todos os dias.

0 desenvolvimento de novos produtos, sejam eles automotivos ou de qualquer outro segmento, é complexo, envolvendo diversas etapas que devem ser planejadas, implementadas e controladas de forma a minimizar os riscos de insucesso (QUINTELLA; ROCHA; ALVES, 2005). 
Essa forte influência e a complexidade inerente desses setores geram a necessidade de adoção de um processo sistemático de desenvolvimento de produto (PDP) pois, segundo Lagrosen (2005), o sucesso de uma empresa industrial é dependente de sua habilidade de desenvolver novos produtos, novos mercados e novas possibilidades de crescimento.

Segundo Toledo et al. (2008), ocorreu uma reestruturação na indústria brasileira de autopeças na década de 1990: ela passou a ser composta por filiais de empresas transnacionais e hoje representa um importante segmento na produção industrial do país.

Esse tipo de indústria fornece seus produtos para grandes montadoras que atuam no Brasil ou no exterior e, por isso, sofre fortes influências no que diz respeito às práticas de gestão do PDP, que devem seguir padrões de qualidade exigidos pelas indústrias do segmento automobilístico.

De acordo com Rozenfeld et al. (2006), empresas que utilizam o PDP de forma sistemática, além de obterem qualidade de produto e processo, alcançam também sucesso em outros fatores de vantagem competitiva como custo, velocidade e confiabilidade de entrega e flexibilidade.

A utilização de um modelo de referência pode auxiliar as empresas no gerenciamento do PDP, em que uma sequência de etapas é definida e padronizada para todos os tipos de produto que forem desenvolvidos. Existem vários modelos na literatura que basicamente se apresentam muito similares, com algumas pequenas diferenças, conforme a visão de cada autor. Dentre os modelos de PDP, alguns focam mais na gestão do processo de desenvolvimento, apesar de caracterizarem também o projeto do produto, tais como os modelos de Wheelwright e Clark (1992), Rosenthal (1992), Cooper (1993), Pahl et al. (2005), Rozenfeld et al. (2006) e Back et al. (2008).

Em vista disso, a questão norteadora deste trabalho é: qual desses seis modelos é mais apropriado para analisar o processo de desenvolvimento de produtos de uma indústria do setor de autopeças?

Sendo assim, o objetivo deste artigo é selecionar um modelo de referência para o processo de desenvolvimento de produtos de uma empresa do ramo de autopeças através de um método de auxílio à decisão por multicritério denominado Analytic Hierarchy Process (AHP). 0 método de pesquisa utilizado foi a modelagem, empregando seis alternativas, três critérios e o julgamento de cinco especialistas.

No Brasil, as pesquisas de campo sobre a indústria automobilística e a indústria de autopeças normalmente focam na gestão e organização da manufatura e nas estratégias e relações com os clientes e fornecedores (cadeia de suprimentos), determinantes de competitividade e outras áreas (RACHID; BRESCIANI FILHO; GITAHY, 2001; SILVEIRA; DINIZ, 2002; POPADIUK et al., 2005; QUINTELLA; ROCHA; ALVES, 2005; MESQUITA; LAZZARINI; CRONIN, 2007; PIRES; SACOMANO NETO, 2008).

Pouco se conhece sobre as práticas de gestão do processo de desenvolvimento de produtos das empresas dessa indústria no país (TOLEDO et al., 2008). Algumas pesquisas focam nas práticas de gestão no PDP (SALERNO et al., 2002; MIGUEL, 2006; QUINTELLA; ROCHA, 2006; IBUSUKI; KAMINSKI, 2007; TOLEDO et al., 2008), mas em se tratando de pesquisas sobre modelos de referências específicos para o PDP dos setores automotivo e de autopeças, pode-se dizer que essas ainda são incipientes.

\section{Método de pesquisa}

Para que os objetivos desta pesquisa sejam alcançados é necessário seguir um método para aplicação do AHP onde, segundo Saaty (1991), são realizados julgamentos quantitativos para selecionar uma melhor alternativa em relação a alguns critérios de decisão.

Diante dessa premissa, a presente pesquisa pode ser classificada em aplicada, de acordo com Marconi e Lakatos (1999), caracterizando-se pela solução de problemas específicos através de aplicações práticas. Quanto à forma de abordar o problema, classifica-se como quantitativa e quanto aos objetivos são explicativos, pois baseiam-se em uma lógica dedutiva que busca explicar relações de causa e efeito entre as alternativas. Em relação ao método utilizou-se a modelagem, conforme Bertrand e Fransoo (2002), em que as etapas de aplicação do AHP foram estruturadas através do software Expert Choice ${ }^{\circledR}$ versão 11.

A coleta de dados se deu por meio de entrevistas semiestruturadas com cinco especialistas da empresa objeto de estudo para a determinação dos critérios de utilização do método de apoio à decisão adotado. A forma como os especialistas participaram da pesquisa está descrita em Descrição da pesquisa e discussão.

\section{Processo de desenvolvimento de produtos}

A competição entre empresas de diversos segmentos tem impulsionado a criação e o desenvolvimento de novos produtos, bem como aperfeiçoamentos em produtos existentes ou reposicionamento de produtos em novos mercados, mostrando diferentes estratégias de projeto (MELLO, 2005). 
0 produto é resultado de um processo sistemático que uma empresa desenvolve para adquirir vantagem competitiva. Um dos principais requisitos que ajudam a garantir essa vantagem competitiva é a criação de um produto com características que satisfaçam às necessidades, sonhos, desejos, valores e expectativas dos clientes.

Chao e Kavadias (2008) definem produto como conjunto de atributos de tecnologia e mercado. Esses mesmos autores afirmam que os atributos representam parâmetros-chave do produto, como arquitetura principal, tecnologias de componentes, características de projetos e especificações do processo de manufatura.

Empresas que definem recursos e executam projetos de desenvolvimento de novos produtos mais eficazmente e eficientemente do que seus competidores são recompensadas com significantes vantagens estratégicas (WHEELWRIGHT; CLARK, 1994).

0 processo de tomada de decisão sobre a maneira como o produto será desenvolvido difere tanto entre as empresas quanto dentro de cada empresa. Diferentes organizações farão diferentes escolhas e podem usar diferentes métodos, mas todas elas tomam decisões sobre uma variedade de questões tais como o conceito do produto, sua arquitetura, configuração, arranjos de aquisição e distribuição, cronograma do projeto etc. (KRISHAN; ULRICH, 2001).

Segundo Amaral e Rozenfeld (2008), processo é qualquer atividade ou conjunto de atividades que utiliza uma entrada, adiciona valor a ela e fornece uma saída (resultado) a um cliente específico. $\mathrm{Na}$ visão de processos, o desenvolvimento de produtos engloba o conjunto de atividades realizadas pelos diversos setores funcionais da empresa, que permitem a transformação de informações sobre necessidades de mercado em informações e recursos para a produção de um produto específico (AMARAL, 2002).

0 PDP consiste num conjunto de atividades por meio das quais se busca, a partir das necessidades do mercado e das possibilidades e restrições tecnológicas e considerando as estratégias competitivas e de produto da empresa, chegar às especificações de projeto de um produto e de seu processo de produção, para que a manufatura seja capaz de produzi-lo (ROZENFELD, 2006).

A gestão do PDP é complexa e se relaciona com todas as demais atividades da empresa (MUNDIM et al., 2002; TOLEDO et al., 2008). lsso exige a integração entre as áreas funcionais através da formação de equipes multidisciplinares para uma troca de informações eficiente entre as etapas de desenvolvimento. Segundo Kohn (2006), quanto mais complexo for o grau de trabalho, mais complexas serão as interdependências entre os departamentos funcionais durante as diferentes fases de desenvolvimento do produto.
Após analisar os principais conceitos abordados sobre o PDP, a próxima seção explora o cenário da indústria de autopeças, bem como as pesquisas científicas realizadas nesse setor de atuação.

\section{Cenário da indústria de autopeças no Brasil}

A abertura da economia brasileira, no início da década de 1990, aliada a uma série de políticas direcionadas para o setor automotivo contribuíram para reestruturação das montadoras de automóveis instaladas no país, especialmente nas suas estratégias de desenvolvimento de produtos (DP). 0 mercado brasileiro, então dominado por Volkswagen, Fiat, Ford e General Motors, incorporou diversas novas montadoras que estabeleceram unidades produtivas no país, como Toyota, Honda, Renault, Peugeot, Citroën, Audi, Nissan, Land Rover, Mitsubishi e Mercedes. lsso intensificou o lançamento de veículos no Brasil na década de 90, que foi três vezes maior do que o observado nos anos 1980 (CONSONI, 2004).

Nesse contexto, as empresas do setor de autopeças recebem forte influência das montadoras, sendo levadas a se reestruturar, tendendo a não desenvolver produtos exclusivos e sim acompanhar os projetos de seus clientes. A reestruturação setorial foi caracterizada pela saída de empresas pequenas e frágeis do mercado e pela realização de fusões a aquisições entre as médias e grandes empresas, o que ainda vem ocorrendo em ritmo reduzido (TOLED0 et al., 2008).

De acordo com o Sindicato Nacional da Indústria de Componentes para Veículos Automotores (2009), existem 648 unidades industriais de autopeças no Brasil, sendo que 496 são associadas a esse órgão sindical. Essas empresas têm intensificado o seu crescimento anualmente, os maiores destinos dos 7,5 bilhões de dólares de exportações têm sido países como Argentina, Estados Unidos, Alemanha e México.

Segundo o Sindipeças (SINDICATO..., 2009), a indústria de autopeças no Brasil representa 5\% do PIB, com investimentos da ordem de 1,4 bilhão de dólares, contribuindo com 197 mil empregos diretos e gerando um faturamento de 24,2 bilhões de dólares. Esse segmento é, em grande parte, internacionalizado, composto na sua totalidade por empresas multinacionais de capital estrangeiro. 0 capital estrangeiro em 1994 era de 48\% e passou para 81\% em 2007.

Algumas pesquisas sobre PDP têm sido desenvolvidas na indústria automotiva no Brasil. Miguel (2006) examinou as atividades de desenvolvimento de produto no Brasil considerando a perspectiva de montadoras e empresas de autopeças. Através de 
uma pesquisa de levantamento, Toledo et al. (2008) analisaram as práticas e tendências de gestão do PDP em empresas da indústria brasileira de autopeças. Esse estudo revelou um crescimento da adoção de modelos de referência para estruturação e gestão do PDP pelas empresas do setor, mas com implementação parcial. Entretanto, nenhuma dessas pesquisas visou utilizar um método de apoio à decisão multicritérios para a seleção do modelo de referência mais indicado para uma empresa do setor. Essa é a contribuição esperada do presente trabalho.

\section{Modelos de referência para desenvolvimento de produtos}

Para Davila (2000), o desenvolvimento de produtos é um processo incerto, por isso precisa ser estruturado através de fases bem definidas, que terminem com uma tomada de decisão sobre o futuro do projeto do novo produto. De acordo com Pahl et al. (2005), uma metodologia de projeto é um procedimento planejado de ações a serem realizadas durante o desenvolvimento de sistemas técnicos.

Essas fases representam o processo de negócio como um fluxo contínuo de atividades, com entradas e saídas bem distintas que determinam o sucesso ou o insucesso da empresa no lançamento de um produto no mercado. Segundo Bremer e Lenza (2000), pelos processos de negócio podem-se materializar as políticas gerenciais, os fluxos de documentos e informações, os procedimentos operacionais e os processos de manufatura.

As fases do processo de desenvolvimento são agrupadas em modelos que, de acordo com Amaral (2002), podem ser divididos em:

- Modelos de referência: são elaborados a partir das melhores práticas do PDP e representam uma aplicação mais ampla e geral que pode ser utilizada como referência para o desenvolvimento de modelos específicos (WHEELWRIGHT; CLARK, 1992; ROSENTHAL, 1992; COOPER, 1993; PAHL et al., 2005; ROZENFELD et al., 2006; BACK et al., 2008);

- Modelos específicos: são modelos que representam e/ou são utilizados por uma organização através de um processo de adaptação (ROMANO, 2003; BARBALHO, 2006; EDGETT, 1996; GLEN; LORD, 1996; GUPTA; PAVAR; SMART, 2007).

Nos modelos específicos, na maioria das pesquisas identificadas, a escolha do modelo de referência a ser usado para a adaptação a um segmento de mercado partiu da escolha do próprio pesquisador, o que de certa forma pode introduzir tendenciosidade ao processo. A utilização de um método de apoio à decisão multicritério para a seleção desse modelo tende a reduzir (ou eliminar) essa tendenciosidade, uma vez que a decisão fica a cargo de especialistas e os critérios adotados são avaliados quanto à sua consistência.

Para que o desenvolvimento de produtos possa cumprir com o seu papel de contribuir com a competitividade da empresa, de acordo com Rozenfeld et al. (2006), um processo eficaz e eficiente de gestão necessita ser implementado com base em um modelo de referência que determine a capacidade de as empresas controlarem o processo de desenvolvimento e de aperfeiçoamento dos produtos e de interagirem com o mercado e com as fontes de inovação tecnológica.

0 modelo de referência contribui para que as empresas passem a executar um processo de desenvolvimento de produtos mais formal e sistemático, integrado aos demais processos empresariais, com os participantes da cadeia de fornecimento e com os clientes finais. Fornece, ainda, os meios para que as empresas inovem e desenvolvam, dentro de suas fábricas, novos produtos (BACK et al., 2008).

Através de um estudo bibliográfico, Suarez, Jung e Caten (2009) identificaram vários modelos de desenvolvimento de produto (DP) e modelos de processos de desenvolvimento de produto (PDP) elaborados por diversos autores, contextualizando-os por marcos históricos da ciência e tecnologia que podem ter influenciado sua concepção, como mostra a Figura 1.

Dentre os modelos de DP e PDP foram escolhidos seis modelos de PDP apresentados na Figura 1, devido ao fato de focarem mais na gestão do processo de desenvolvimento, apesar de caracterizarem também o projeto do produto. São os seguintes autores: Wheelwright e Clark (1992), Rosenthal (1992), Cooper (1993), Pahl et al. (2005), Rozenfeld et al. (2006) e Back et al. (2008).

Posteriormente neste trabalho será utilizado o AHP para determinar dentre esses seis modelos selecionados qual o mais indicado para analisar o processo de desenvolvimento de produtos da empresa objeto de estudo.

\section{Método de auxílio à decisão por múltiplos critérios}

Existem vários métodos de auxílio à decisão por múltiplos critérios mas, de acordo com Salomon (2004), os métodos mais utilizados em pesquisas são: AHP (Analytical Hierarchy Process), ELECTRE (Elimination et Choix Traduisant la Réalité) e MACBETH (Measuring Attractiveness by a Categorical Based Evaluation Technique). Existe outro método conhecido como ANP (Analytical Network Process) que avalia a 


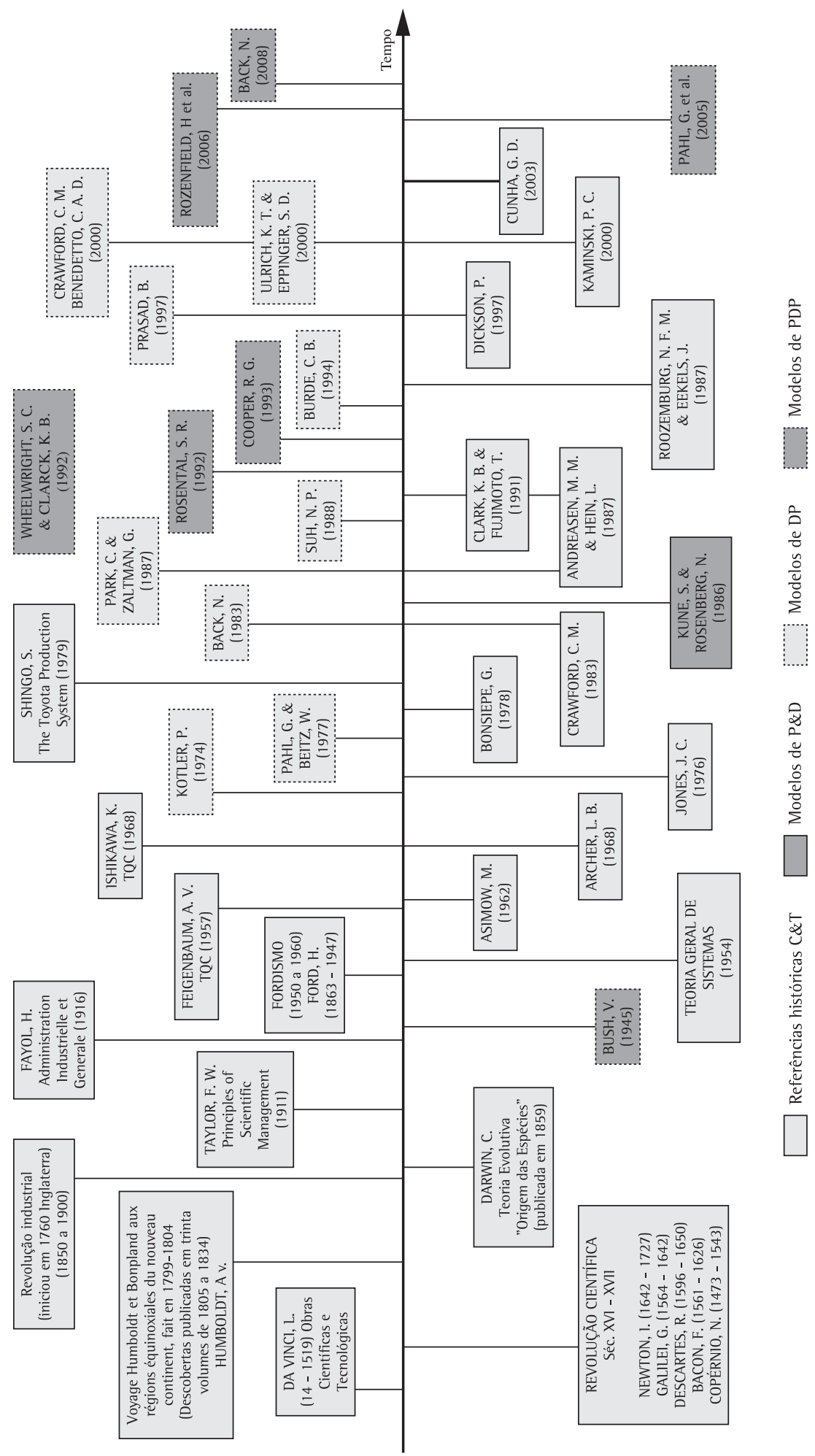

Figura 1. Modelos de referência de desenvolvimento de produtos contextualizados por marcos históricos da ciência e tecnologia. Fonte: adaptado de Suarez, Jung e Caten (2009). 
dependência entre critérios e alternativas da hierarquia proposta por Saaty (2008). De acordo com Salomon, Montevechi e Pamplona (1999), o AHP tem preferência sobre os outros métodos de decisão multicritérios desde que se disponha de tempo para a tomada de decisão, existam no máximo nove alternativas e que essas alternativas e os critérios sejam independentes.

A justificativa para escolha do AHP na tomada de decisão é a sua extensa aplicabilidade, simplicidade, facilidade de uso e ótima flexibilidade (HO, 2008). Segundo o mesmo autor, esse método pode ser integrado com outras técnicas, por exemplo, programação matemática, QFD (Quality Function Deployment), análise SWOT (Strengths, Weaknesses, Opportunities e Threats) e DEA (Data Envelopment Analysis), tornando-o mais realista por considerar não somente fatores qualitativos e quantitativos, mas também limitações de recursos existentes nas organizações.

Outras vantagens na utilização do AHP que podem-se destacar: a não necessidade de um software proprietário (SALOMON, 2004), exigência de um menor número de julgamentos em relação ao ANP (SALOMON; MONTEVECHI, 1997) e análise de consistência das comparações, mostrando os julgamentos inconsistentes (CHENG; Ll, 2003). Por essas razões, adotou-se neste trabalho o AHP para seleção do modelo de referência na empresa objeto de estudo.

Para Ho (2008), o AHP apresenta três operações principais: construção da hierarquia, análise de prioridade e verificação da consistência. Desenvolvido por Thomas L. Saaty na década de 1970, o AHP é, segundo Saaty (2008), uma teoria de medição relativa baseada na comparação entre pares de julgamentos de especialistas para obter uma escala de prioridades. As comparações são feitas usando uma escala de julgamentos absolutos que representa o quanto uma determinada alternativa estabelece dominância sobre outra em relação a um determinado critério.

0 AHP é um método de auxílio à decisão por múltiplos critérios baseado na quebra da complexidade do problema pela decomposição hierárquica em partes menores, ou variáveis, homogeneamente agrupadas (objetivo, critérios, subcritérios e alternativas); seguida da mensuração qualitativa (julgamento subjetivo) ou quantitativa da importância relativa dessas variáveis, em cada nível da hierarquia, por meio de comparações pareadas, transportadas para valores numéricos de uma escala de razão em matrizes recíprocas positivas; a partir dessas, o problema sofre um processo de síntese matemática pela derivação de vetores de prioridade normalizados, os quais, de forma aditiva no sentido inverso da decomposição hierárquica, expressam a ordem das alternativas frente ao objetivo (GUIMARÃES, 2007).
0 método tem sido amplamente utilizado em diversas áreas de aplicação por diversos pesquisadores para seleção de alternativas baseadas em critérios pré-definidos e resolução de problemas na tomada de decisões, que deixaram de ser subjetivas e arbitrárias para serem objetivas e mais realistas. Guimarães (2007) utilizou o AHP para selecionar um equipamento médico-hospitalar, Al-Harbi (2001) introduziu a utilização do método com gerenciamento de projetos, Hsiao (2002) integrou as técnicas de QFD, FMEA e DFA com o AHP para desenvolver um projeto de produto com alta qualidade e menores custos. Muller e Fairlie-Clarke (2001) utilizaram o AHP para determinar os fatores de correlação na estimativa do impacto na lucratividade das várias questões do produto a serem tratadas pela empresa durante o PDP. Hsu e Chen (2008) fizeram uso do AHP para selecionar a mais apropriada franquia de lojas de bens duráveis. Rosa, Sellito e Mendes (2006) separaram fornecedores em aglomerados através da aplicação do AHP no setor de compras.

Em relação à seleção de modelos para o processo de desenvolvimento de produtos e seleção de abordagens de gestão do conhecimento, Fass et al. (2009) aplicaram o AHP para selecionar o modelo de PDP mais adequado para empresas de base tecnológica e Neves et al. (2009) utilizaram esse mesmo método para selecionar uma abordagem de gestão do conhecimento que atendesse aos critérios aplicáveis à tomada de decisão e gerenciamento de riscos em projetos de empresas de base tecnológica. Diante dessa contextualização, a presente pesquisa se baseia na seleção de um modelo de referência do PDP para uma indústria de autopeças. As etapas de aplicação do método são detalhadas nos tópicos a seguir.

\section{Descrição da pesquisa e discussão}

A empresa selecionada como objeto de estudo é uma multinacional alemã do ramo de autopeças que fornece sistemas e componentes para a indústria automotiva enviando seus produtos para as mais conceituadas montadoras no mercado nacional e de exportação.

Com 110 instalações em quatro continentes que empregam mais de 30 mil pessoas, a empresa tem intensificado o seu crescimento nos últimos anos para aumentar sua competitividade por meio de inovações tecnológicas no desenvolvimento de produtos, contando com oito centros de pesquisa e desenvolvimento espalhados pelo mundo.

A unidade de análise desta pesquisa localiza-se em Itajubá, no sul de Minas Gerais, e fabrica anéis de pistão, buchas e arruelas. Nela foram selecionados os especialistas que realizaram os julgamentos do AHP. 
0 processo de desenvolvimento de produtos (PDP) da empresa é baseado na VDA 6.0, norma de qualidade para fornecedores da indústria automotiva alemã; na ISO/TS 16949, norma de padrões de qualidade exigida para fornecedores da indústria automotiva em geral, que engloba requisitos de todas as outras normas; e também em um procedimento interno alicerçado no gerenciamento de projetos para assegurar um desenvolvimento do produto padronizado.

0 objetivo básico do PDP da empresa é o desenvolvimento de um produto específico pronto para produção em série. O PDP define todas as funções envolvidas no processo de desenvolvimento, incluindo tarefas, responsabilidades e interfaces claras e objetivas que auxiliam a equipe de desenvolvimento a alcançar os objetivos estratégicos da empresa que são alta qualidade e redução do tempo e dos custos de desenvolvimento.

A necessidade de a empresa de criar um novo produto surge quando uma indústria automobilística está projetando um novo motor e precisa desenvolver fornecedores de peças que garantam as características de funcionamento especificadas para esse motor, tais como potência, consumo, controle de emissões de gases na atmosfera, custo etc.

De Paula e Mello (2009) realizaram uma análise comparativa entre quatro modelos de referência de PDP teóricos com o modelo de PDP utilizado pela empresa objeto de estudo. Os modelos teóricos foram: Wheelwright e Clark (1992), Cooper (1993), Pahl et al. (2005) e Rozenfeld et al. (2006). A essa comparação foram acrescentados os modelos de Rosenthal (1992) e Back et al. (2008), gerando as informações dadas pelo Quadro 1.

Esses seis modelos de referência são as alternativas a serem inseridas na estrutura do AHP. Para facilitar a inserção dessas informações no software Expert Choice, cada modelo foi abreviado através de siglas, como mostra o Quadro 2.

Depois de definidas as alternativas, foram definidos os critérios a serem usados para selecionar as alternativas disponíveis. A determinação dos critérios foi baseada em entrevistas com cinco especialistas que atuam há mais de três anos diretamente no PDP

Quadro 1. Comparação das fases dos modelos teóricos com as do modelo da empresa.

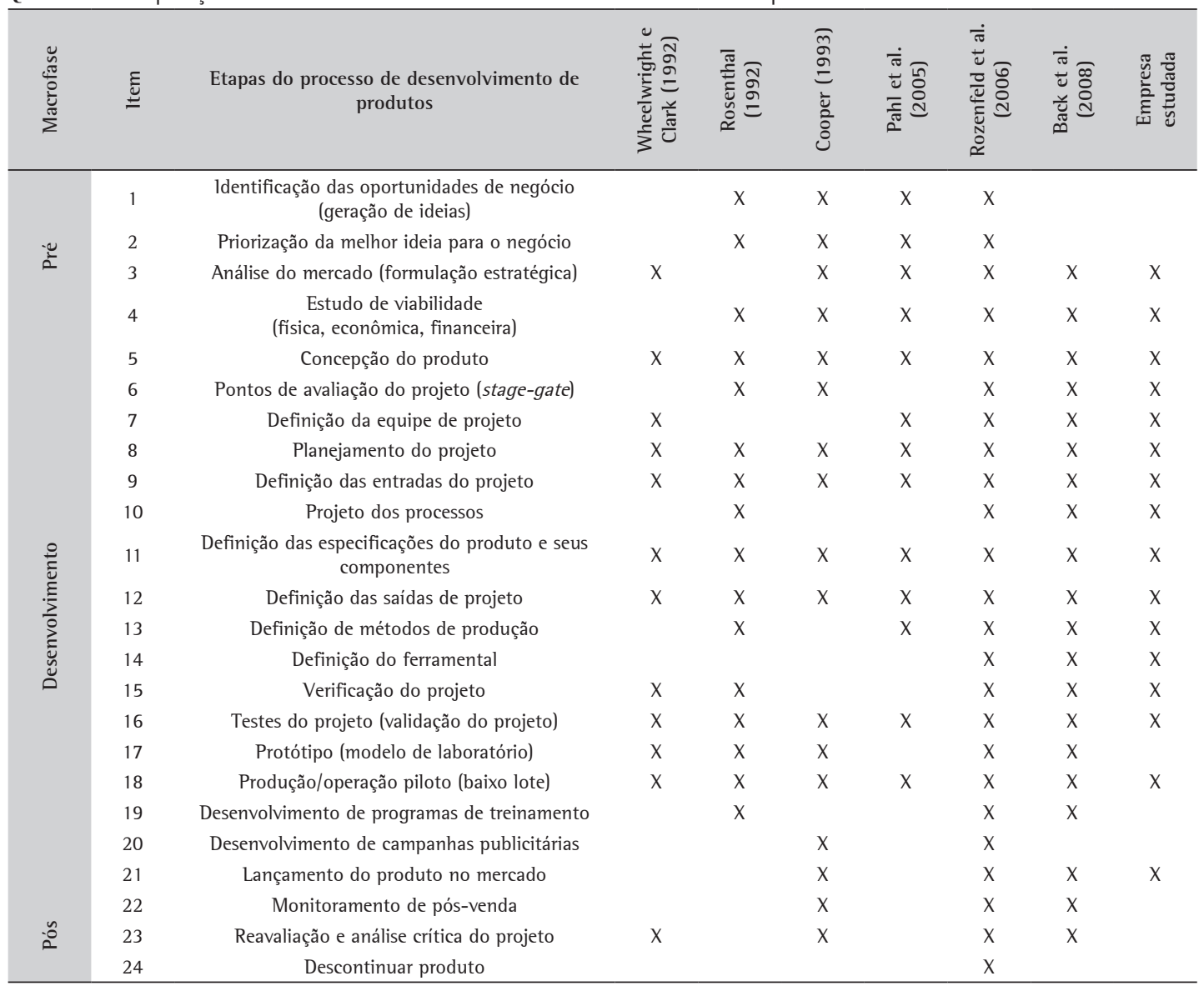


da empresa (vide Quadro 3) e em artigos publicados que caracterizam o PDP de indústrias de autopeças. Os especialistas contribuíram com sua experiência no tema e nos processos da empresa para a definição dos critérios. Os critérios adotados foram:

- Inovação: inovação na indústria através da criação e desenvolvimento de produtos;

- Integração dos processos: integração do PDP com outros processos da empresa, com os participantes da cadeia de fornecimento e com os clientes finais;

- Sistematização do PDP: formalização e sistematização do PDP, ferramentas de apoio que o modelo propõe ao processo de tomada de decisão.

Com base nas informações das alternativas e dos critérios, foi construída a hierarquia do AHP da Figura 2 que, segundo Saaty (2008), pode ser estruturada em uma árvore de decisão, dividindo-se esta em níveis, colocando-se no primeiro nível, o objetivo, nos níveis

Quadro 2. Abreviação dos modelos de referência.

\begin{tabular}{cc}
\hline Modelos de referência & Abreviação \\
\hline Pahl et al. (2005) & PAH \\
Wheelwright e Clark (1992) & WCL \\
Rosenthal (1992) & ROS \\
Cooper (1993) & COO \\
Back et al.(2008) & BAC \\
Rozenfeld et al. (2006) & ROZ \\
\hline
\end{tabular}

Quadro 3. Especialistas selecionados para realizar os julgamentos.

\begin{tabular}{ccc}
\hline Qtde. & Função & $\begin{array}{c}\text { Experiência em } \\
\text { PDP }\end{array}$ \\
\hline 1 & Gerente de planejamento de produto & mais de 10 anos \\
1 & Chefe de planejamento de produto & 8 anos \\
1 & Engenheiro de planejamento de & produto \\
& Analistas de planejamento de \\
produto & 3 anos \\
\hline
\end{tabular}

intermediários, os critérios e os subcritérios, e nos menores níveis, as alternativas.

Os especialistas da empresa participaram de um seminário de 15 horas sobre os seis modelos estudados, ministrado pelo pesquisador, onde foram detalhados os principais conceitos, diferenças e fases propostas pelos autores de cada modelo de referência, bem como as atividades de casa fase que auxiliam na análise do PDP da empresa. Isso serviu para informar os especialistas sobre o processo, vantagens e desvantagens de cada um dos seis modelos selecionados na literatura. Esses seis modelos foram escolhidos por serem os mais referenciados na literatura pesquisada.

Após conhecerem os modelos, os especialistas realizaram os julgamentos entre critérios e também entre as alternativas em relação a cada critério, resultando em uma matriz pareada dos elementos da hierarquia. 0 pesquisador intermediou esse processo nas dependências da empresa, mostrando aos especialistas como preencher os dados no software utilizado, tomando o cuidado de não influenciar nas decisões dos especialistas, o que prejudicaria os resultados da presente pesquisa.

A escala utilizada nos julgamentos é a proposta por Saaty (1991), composta por números absolutos de 1 a 9, como mostra o Quadro 4. De acordo com Al-Harbi (2001), as matrizes são preenchidas com $n(n-1)$ julgamentos onde $n$ é a ordem da matriz.

Em seguida foi realizada a combinação de todos os julgamentos realizados pelos cinco especialistas através da média geométrica dos julgamentos para se chegar nos vetores de prioridade geral $V$ e também nos pesos de priorização dos critérios e das alternativas $W$, conforme proposto por Saaty e Shang (2007). A partir dessa fase da pesquisa, a condução das análises por meio do método de apoio à decisão multicriterial foi realizada apenas pelo pesquisador, sem a participação dos especialistas. Isso foi intencional, uma vez que

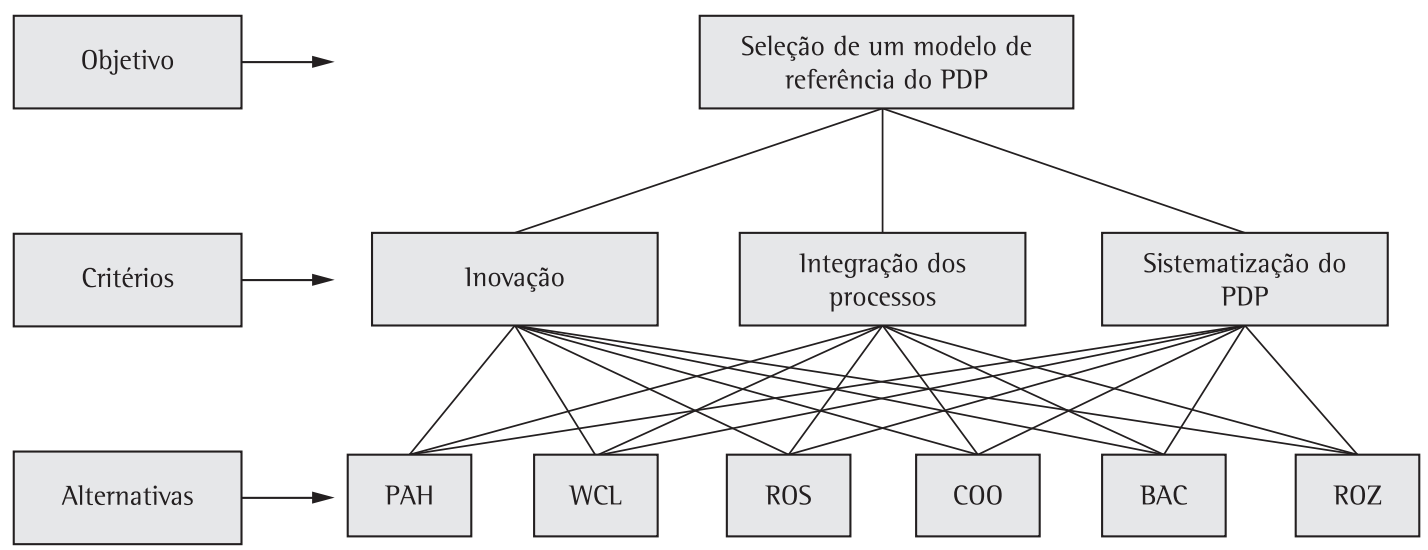

Figura 2. Estrutura hierárquica do AHP para a presente pesquisa. 
visava-se utilizar uma das potencialidades do método de minimizar a tendenciosidade no processo de seleção do modelo de referência (uma vez feitos os julgamentos, apenas a formulação matemática iria indicar o modelo mais apropriado para os critérios selecionados).

Existem outros dois métodos para realizar a combinação dos julgamentos. Um deles, de acordo com Saaty (2003), é quando surge a necessidade de agrupar os julgamentos via autovetor, ou seja, quando os julgamentos entre os indivíduos que compõem determinado grupo são dispersos. Para Saaty e Shang (2007), o outro método é realizado pela votação consenso, quando a tomada de decisão precisa ser realizada em grupo e se faz necessário agregar as preferências dos indivíduos em uma classificação consenso, o que exige que o grupo entre em acordo sobre cada entrada da matriz de julgamento.

De acordo com Al-Harbi (2001), depois da construção das matrizes pareadas dos critérios e alternativas é necessário avaliar a consistência dos julgamentos dos especialistas, o que Saaty (1991) denominou como razão de consistência (CR), determinada pela Equação 1.

$$
C R=\frac{C l}{R l}
$$

Onde $\mathrm{Cl}$ é chamado de índice de consistência, representado pela Equação 2, e Rl é o índice randômico médio determinado estatisticamente pelo Oak Ridge National Laboratory (SAATY, 1991), podendo ser selecionado conforme a ordem $n$ das matrizes dos julgamentos na Tabela 1.

$$
C l=\frac{\lambda_{\max }-n}{(n-1)}
$$

Onde $\lambda_{\text {max }}$ é o autovalor encontrado na matriz de comparação pareada dos critérios e das alternativas e $n$ representa a ordem da matriz.

Segundo Hsiao (2002), se o valor do CR for superior a 10\%, os julgamentos estão inconsistentes e devem ser revisados. No presente trabalho, os julgamentos dos critérios e das alternativas em relação a cada critério foram consistentes, pois o CR calculado em cada matriz apresentou um valor inferior a 10\%.

A Tabela 2 mostra a matriz de comparação dos critérios com os respectivos pesos relativos.

0 critério inovação apresentou uma diferença significativa em relação aos critérios integração do processo e sistematização do PDP, assumindo um peso relativo de $67,82 \%$ na hierarquia do AHP. É interessante ressaltar que a empresa investe constantemente no desenvolvimento de seus produtos, criando inovações tecnológicas para atender às necessidades de seus clientes. Em segundo lugar aparece o critério sistematização do PDP, com 16,46\%, e em terceiro, o critério integração do processo, com 15,72\%.

\begin{tabular}{|c|c|c|}
\hline $\begin{array}{l}\text { Intensidade de } \\
\text { importância }\end{array}$ & Definição & Explicação \\
\hline 1 & Mesma importância & As duas atividades contribuem igualmente para o objetivo \\
\hline 3 & Importância pequena de uma sobre a outra & $\begin{array}{c}\text { A experiência e o julgamento favorecem levemente uma } \\
\text { atividade em relação a outra }\end{array}$ \\
\hline 5 & Importância grande ou essencial & $\begin{array}{c}\text { A experiência e o julgamento favorecem fortemente uma } \\
\text { atividade em relação a outra }\end{array}$ \\
\hline 7 & Importância muito grande ou demonstrada & $\begin{array}{c}\text { Uma atividade é muito fortemente favorecida em relação } \\
\text { a outra; sua dominação de importância é demonstrada na } \\
\text { prática }\end{array}$ \\
\hline 9 & Importância absoluta & $\begin{array}{c}\text { A evidência favorece com o mais alto grau de certeza uma } \\
\text { atividade em relação a outra }\end{array}$ \\
\hline $2,4,6,8$ & Valores intermediários entre os valores adjacentes & $\begin{array}{l}\text { Quando se procura uma condição de compromisso entre duas } \\
\text { definições }\end{array}$ \\
\hline $\begin{array}{l}\text { Recíprocos dos valores } \\
\text { acima de zero }\end{array}$ & $\begin{array}{c}\text { Se uma atividade } i \text { recebe uma das designações } \\
\text { diferentes acima de zero quando comparada com } \\
\text { a atividade } j \text {, então } j \text { tem um valor recíproco } \\
\text { quando comparado com } i\end{array}$ & Uma designação razoável \\
\hline Racionais & Razões resultantes da escala & $\begin{array}{c}\text { Se a consistência tiver de ser forçada para obter valores } \\
\text { numéricos n para completar a matriz }\end{array}$ \\
\hline
\end{tabular}

Quadro 4. Escala fundamental de Saaty.

Fonte: Saaty (1991).

\begin{tabular}{|c|c|c|c|c|c|c|c|c|c|c|c|c|c|}
\hline $\mathrm{n}$ & 1 & 2 & 3 & 4 & 5 & 6 & 7 & 8 & 9 & 10 & 11 & 12 & 13 \\
\hline $\mathrm{Rl}$ & 0,00 & 0,00 & 0,58 & 0,90 & 1,12 & 1,24 & 1,32 & 1,41 & 1,45 & 1,49 & 1,51 & 1,48 & 1,56 \\
\hline
\end{tabular}

Tabela 1. Índice randômico do AHP.

Fonte: Saaty (1991) 
As Tabelas 3, 4 e 5 mostram a matriz de às outras, obtendo o resultado final do AHP. Esse comparação dos modelos de PDP em relação a cada critério, com os respectivos pesos relativos.

A multiplicação dos pesos (W) relativos dos critérios da Tabela 2, pelos pesos (W) relativos a cada alternativa disponíveis nas Tabelas 3, 4 e 5 resulta no vetor de prioridade geral, ou seja, o vetor de decisão que ordena a prioridade de uma alternativa em relação

\section{resultado pode ser visto na Tabela 6 .}

Baseado nas informações da Tabela 6, o modelo selecionado pelo AHP a ser utilizado na análise do processo de desenvolvimento de produtos dessa empresa do setor de autopeças é o de Rozenfeld et al. (2006), que aparece em primeiro lugar, com um peso na hierarquia de $39,26 \%$.

Tabela 2. Matriz de julgamento e priorização dos critérios.

\begin{tabular}{|c|c|c|c|c|c|}
\hline & Inovação & $\begin{array}{l}\text { Integração do } \\
\text { processo }\end{array}$ & $\begin{array}{c}\text { Sistematização do } \\
\text { PDP }\end{array}$ & Autor vetor (V) & Pesos (W) \\
\hline lnovação & 1 & 4,514 & 3,936 & 2,610 & $67,82 \%$ \\
\hline Integração do processo & 0,222 & 1 & 1 & 0,605 & $15,72 \%$ \\
\hline Sistematização do PDP & 0,254 & 1 & 1 & 0,633 & $16,46 \%$ \\
\hline \multicolumn{6}{|l|}{$\lambda \max =3,0021$} \\
\hline $\mathrm{CR}=0,18 \%$ & & & & & \\
\hline
\end{tabular}

Tabela 3. Matriz de julgamento dos modelos para o critério inovação.

\begin{tabular}{|c|c|c|c|c|c|c|c|c|}
\hline lnovação & РАH & WCL & ROS & COO & BAC & ROZ & $\begin{array}{l}\text { Autor vetor } \\
\text { (V) }\end{array}$ & Pesos (W) \\
\hline РAH & 1 & 0,608 & 0,242 & 0,237 & 0,172 & 0,140 & 0,307 & $3,76 \%$ \\
\hline WCL & 1,644 & 1 & 0,370 & 0,412 & 0,278 & 0,206 & 0,493 & $6,04 \%$ \\
\hline ROS & 4,129 & 2,702 & 1 & 0,871 & 0,375 & 0,226 & 0,968 & $11,85 \%$ \\
\hline $\mathrm{COO}$ & 4,227 & 2,426 & 1,149 & 1 & 0,308 & 0,226 & 0,968 & $11,85 \%$ \\
\hline BAC & 5,827 & 3,594 & 2,667 & 3,245 & 1 & 0,530 & 2,140 & $26,19 \%$ \\
\hline ROZ & 7,137 & 4,856 & 4,416 & 4,416 & 1,888 & 1 & 3,294 & $40,31 \%$ \\
\hline \multicolumn{9}{|c|}{$\lambda \max =6,1821$} \\
\hline \multicolumn{9}{|c|}{$\mathrm{CR}=2,94 \%$} \\
\hline
\end{tabular}

Tabela 4. Matriz de julgamento dos modelos para o critério integração do processo.

\begin{tabular}{|c|c|c|c|c|c|c|c|c|}
\hline $\begin{array}{l}\text { Integração do } \\
\text { processo }\end{array}$ & $\mathrm{PAH}$ & WCL & ROS & $\mathrm{COO}$ & BAC & $\mathrm{ROZ}$ & $\begin{array}{l}\text { Autor vetor } \\
\text { (V) }\end{array}$ & Pesos (W) \\
\hline РАН & 1 & 0,608 & 0,326 & 0,361 & 0,157 & 0,144 & 0,343 & $4,31 \%$ \\
\hline WCL & 1,644 & 1 & 0,699 & 0,803 & 0,242 & 0,202 & 0,596 & $7,50 \%$ \\
\hline ROS & 3,064 & 1,431 & 1 & 0,922 & 0,312 & 0,270 & 0,836 & $10,51 \%$ \\
\hline $\mathrm{COO}$ & 2,766 & 1,246 & 1,084 & 1 & 0,291 & 0,268 & 0,814 & $10,24 \%$ \\
\hline $\mathrm{BAC}$ & 6,382 & 4,129 & 3,201 & 3,438 & 1 & 1,084 & 2,608 & $32,79 \%$ \\
\hline ROZ & 6,943 & 4,959 & 3,707 & 3,728 & 0,922 & 1 & 2,757 & $34,66 \%$ \\
\hline \multicolumn{9}{|c|}{$\lambda \max =6,0320$} \\
\hline \multicolumn{9}{|c|}{$\mathrm{CR}=0,52 \%$} \\
\hline
\end{tabular}

Tabela 5. Matriz de julgamento dos modelos para o critério sistematização do PDP.

\begin{tabular}{|c|c|c|c|c|c|c|c|c|}
\hline $\begin{array}{c}\text { Sistematização do } \\
\text { PDP }\end{array}$ & РAH & WCL & ROS & $\mathrm{COO}$ & BAC & $\mathrm{ROZ}$ & $\begin{array}{l}\text { Autor vetor } \\
\text { (V) }\end{array}$ & Pesos (W) \\
\hline РАН & 1 & 0,803 & 0,322 & 0,276 & 0,245 & 0,187 & 0,385 & $5,07 \%$ \\
\hline WCL & 1,246 & 1 & 0,506 & 0,669 & 0,268 & 0,193 & 0,529 & $6,95 \%$ \\
\hline ROS & 3,104 & 1,974 & 1 & 0,871 & 0,467 & 0,281 & 0,943 & $12,39 \%$ \\
\hline $\mathrm{COO}$ & 3,630 & 1,495 & 1,149 & 1 & 0,488 & 0,281 & 0,975 & $12,81 \%$ \\
\hline BAC & 4,076 & 3,728 & 2,141 & 2,048 & 1 & 0,488 & 1,787 & $23,48 \%$ \\
\hline ROZ & 5,335 & 5,194 & 3,554 & 3,554 & 2,048 & 1 & 2,991 & $39,31 \%$ \\
\hline \multicolumn{9}{|c|}{$\lambda \max =6,0850$} \\
\hline \multicolumn{9}{|c|}{$\mathrm{CR}=1,38 \%$} \\
\hline
\end{tabular}


Tabela 6. Classificação final das alternativas.

\begin{tabular}{ccc}
\hline Modelo & $\begin{array}{c}\text { Vetor de decisão } \\
(\%)\end{array}$ & Classificação \\
\hline ROZ & 39,26 & $1^{\circ}$ \\
BAC & 26,78 & $2^{\circ}$ \\
COO & 11,75 & $3^{\circ}$ \\
ROS & 11,73 & $4^{\circ}$ \\
WCL & 6,42 & $5^{\circ}$ \\
PAH & 4,06 & $6^{\circ}$ \\
\hline
\end{tabular}

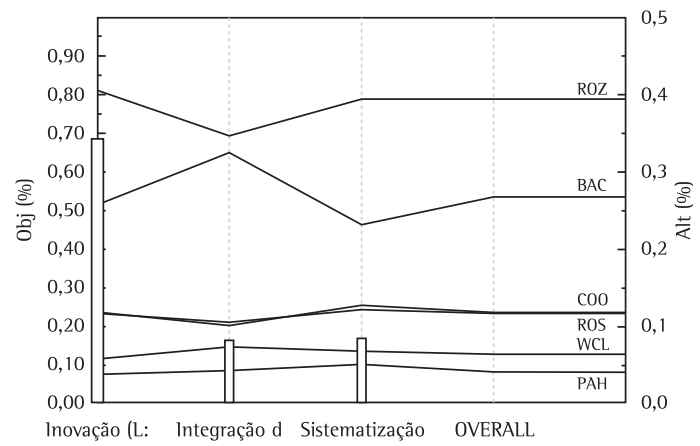

Figura 3. Análise global do AHP.

Para finalizar a aplicação do AHP, realizou-se a análise global dos pesos relativos dos critérios em relação ao objetivo e do desempenho das alternativas com relação aos critérios, dado pela Figura 3.

Peso dos critérios com relação às alternativas apresentou o critério inovação, com 67,82\% do objetivo, em seguida, a sistematização do PDP, com $16,46 \%$, e, por último, a integração do processo, com 15,72\%. Levando em consideração o objetivo proposto, o critério de maior peso foi a inovação. Analisando-se as alternativas com relação aos critérios, o modelo de Rozenfeld et al. (2006) apresentou maior participação em relação aos demais modelos, considerando-se os três critérios de decisão, ou seja, ele é superior em todos os parâmetros disponíveis para realizar a análise do processo de desenvolvimento de produtos da empresa.

\section{Conclusão}

0 presente trabalho atingiu seu objetivo de aplicar um método de apoio à decisão multicritério para a seleção de um modelo de PDP para uma indústria do ramo de autopeças.

A utilização de um método por multicritério para selecionar entre os seis modelos pré-selecionados o que melhor se adequa à realização de uma análise do PDP de uma empresa de autopeças se mostrou relevante, pois contribuiu para a diminuição do subjetivismo, tendenciosidade e arbitrariedade do processo, ao contrário do que se percebeu na literatura pesquisada.
A despeito de alguns críticos que podem considerar que o uso de especialistas que não conhecem os modelos poderia ser considerado um ponto fraco no emprego do método, considera-se que o treinamento ministrado aos mesmos para a realização da pesquisa tenha sido suficiente para sanar tal deficiência. Além disso, como no caso da presente pesquisa, onde o objetivo era identificar um modelo de referência para comparação com a sistemática adotada pelo PDP da empresa, os especialistas selecionados eram as pessoas mais capacitadas para tal, uma vez que trabalhavam na empresa há mais de três anos. Por um lado, não conhecer de antemão os modelos até pode ser considerado vantagem, pois evitou a ocorrência de tendenciosidade por um deles.

Uma sugestão para pesquisa futura é que o modelo de Rozenfeld et al. (2006) seja utilizado para analisar o PDP da empresa onde o AHP foi aplicado, para que cada fase do modelo selecionado possa ser delineada em comparação com as fases de desenvolvimento de produtos da empresa e as melhores práticas de PDP possam ser aplicadas, contribuindo para o levantamento de informações para adaptação de um modelo específico para a indústria de autopeças por futuros pesquisadores.

Outra sugestão para futura pesquisa é que se empreguem métodos de apoio à decisão por multicritérios na seleção de modelos para adaptação para um segmento de mercado específico, assim como foi feito neste trabalho para o caso das indústrias de autopeças. Posteriormente será possível avaliar as vantagens e desvantagens do emprego de cada método para este fim.

\section{Referências}

AL-HARBI, K. M. A. Application of the AHP in project management. International Journal of Project Management, v. 19, n. 1, p. 19-27, 2001. http://dx.doi. org/10.1016/S0263-7863(99)00038-1

AMARAL, D. C. Arquitetura para gerenciamento de conhecimentos explícitos sobre o processo de desenvolvimento de produto. 2002. Tese (Doutorado em Engenharia Mecânica)-Escola Politécnica, Universidade de São Paulo, São Carlos, 2002.

AMARAL, C. S. T.; ROZENFELD, H. Sistematização das melhores práticas de desenvolvimento de produtos para acesso livre e compartilhado na internet. Produto \& Produção, v. 9, n. 2, p. 120-135, 2008.

BACK, N. et al. Projeto integrado de produtos: planejamento, concepção e modelagem. Barueri: Malone, 2008.

BARBALHO, S. C. M. Modelo de referência para o desenvolvimento de produtos mecatrônicos: proposta e aplicações. 2006. Tese (Doutorado em Engenharia Mecânica)-Escola de Engenharia de São Carlos, Universidade de São Paulo, São Carlos, 2006.

BERTRAND, J. W. M.; FRANSO0, J. C. Modeling and simulation: operations management research methodologies using quantitative modeling. 
International Journal of Operations and Production Management, v. 22, n. 2, p. 241-264, 2002. http:// dx.doi.org/10.1108/01443570210414338

BREMER, C. F.; LENZA, R. P. Um modelo de referência para gestão da produção em sistemas de produção assembly to order - ATO e suas múltiplas aplicações. Gestão \& Produção, v. 7, n. 3, p. 269-282, 2000.

CHAO, R. 0.; KAVADIAS, S. A theorical framework for managing the new product development portfolio: when and how to use strategic buckets. Management Science, v. 54, n. 5, p. 907-921, 2008. http://dx.doi.org/10.1287/ mnsc. 1070.0828

CHENG, E. W. L.; Ll, H. Utility of consistency measure in the analytic hierarchy process. Construction Innovation: Information, Process, Management, v. 3, n. 4, p. 231247, 2003.

CONSONI, F. Desenvolvimento de produtos nas montadoras de automóveis no Brasil. Comciência - Revista Eletrônica de Jornalismo Científico, 2004. Disponível em: <http:// www.comciencia.br/200404/reportagens/13.shtml>. Acesso em: abr. 2009.

COOPER, R. G. Winning at new products: accelerating the process from idea to launch. Massachusetts: Addison Wesley Publishing Company, 1993.

DAVILA, T. An empirical study on the drivers of management control systems design in new product development. Account, Organizations and Society, v. 25 , p. 383-409, 2000. http://dx.doi.org/10.1016/ S0361-3682(99)00034-3

DE PAULA, J. 0.; MELLO, C. H. P. Análise comparativa de modelos de PDP: um estudo de caso em uma empresa de autopeças. In: XVI SIMPEP - SIMPÓSIO DE ENGENHARIA DE PRODUÇÃO, 2009, Bauru. Anais... Bauru, 2009, p. 1-12.

EDGETT, S. J. The new product development process for commercial financial services. Industrial Marketing Management, v. 25, n. 6, p. 507-515, 1996. http:// dx.doi.org/10.1016/S0019-8501(96)00067-3

FASS, F. D. M. et al. Seleção de um modelo de processo de desenvolvimento de produto para indústria de base tecnológica do ramo eletroeletrônico. In: ENCONTRO NACIONAL DE ENGENHARIA DE PRODUÇÃO - ENEGEP, 29., 2009, Salvador. Anais... Salvador, 2009. p. 1-13.

GLEN, J. M. W.; LORD, M. New product development processes within the UK medical device industry. Medical Engineering \& Physics, v. 18, n. 8, p. 670-676, 1996. http://dx.doi.org/10.1016/S1350-4533(96)00021-5

GUIMARÃES, í. F. Tomada de decisão com múltiplos critérios na seleção de equipamento médico hospitalar. 2007. Dissertação (Mestrado em Engenharia de Produção)-Universidade Federal de Itajubá, Itajubá, 2007.

GUPTA, A.; PAWAR, K. S.; SMART, P. New product development in the pharmaceutical and telecommunication industries: a comparative study. International Journal of Production Economics, v. 106, n. 1, p. 41-60, 2007. http://dx.doi. org/10.1016/j.ijpe.2006.04.008

HO, W. Integrated analytic hierarchy process and its applications: a literature review. European Journal of Operational Research, v. 186, n. 1, p. 211-228, 2008. http://dx.doi.org/10.1016/j.ejor.2007.01.004

HSIAO, S. W. Concurrent design method for developing a new product. International Journal of Industrial Ergonomics, v. 29 , n. 1, p. 41-55, 2002. http://dx.doi.org/10.1016/ S0169-8141(01)00048-8
HSU, P. F.; CHEN, B. Y. Integrated analytic hierarchy process and entropy to develop a durable goods chain store franchisee selection model. Asia Pacific Journal of Marketing and Logistics, v. 20, n. 1, p. 44-54, 2008. http://dx.doi.org/10.1108/13555850810844869

IBUSUKI, U.; KAMINSKI, P. C. Product development process with focus on value engineering and target-costing: a case study in a automotive company. International Journal Production Economics, v. 105, p. 459-474, 2007. http://dx.doi.org/10.1016/j.ijpe.2005.08.009

KRISHAN, V.; ULRICH, K. T. Product development decisions: a review of the literature. Management Science, v. 47, n. 1, p. 1-21, 2001.

$\mathrm{KOHN}, \mathrm{K}$. Managing the balance of perspectives in the early phase of NPD: a case study from the automotive industry. European Journal of Innovation Management, v. 9, n. 1, p. 44-60, 2006. http://dx.doi. org/10.1108/14601060610640014

LAGROSEN, S. Customer involvement in new product development: a relationship marketing perspective. European Journal of Innovation Management, v. $8, \quad$ n. $4, \quad$ p. 424-436, 2005. http://dx.doi. org/10.1108/14601060510627803

MARCONI, M. A.; LAKATOS, E. M. Técnicas de pesquisa: planejamento e execução de pesquisa, amostragens e técnicas de pesquisas, elaboração, análise e interpelação de dados. São Paulo: Atlas, 1999.

MELlO, C. H. P. Modelo para projeto e desenvolvimento de serviços. 2005. Tese (Doutorado em Engenharia da Produção)-Escola Politécnica, Universidade de São Paulo, São Paulo, 2005.

MESQUITA, L. F.; LAZZARINI, S. G.; CRONIN, P. Determinants of firm competitiveness in Latin American emerging economies: evidence from Brazil's auto-parts industry. International Journal of Operations \& Production Management, v. 27, n. 5, p. 501-523, 2007. http:// dx.doi.org/10.1108/01443570710742384

MIGUEL, P. A. C. The potential of new product development in the automotive industry in Brazil: an exploratory study. Product: Management \& Development, v. 4, n. 1, p. 35-43, 2006.

MULLER, M. H.; FAIRLIE-CLARKE, A. C. Using the AHP to determine the correlation of product issues to profit. European Journal of Marketing, v. 35, n. 7-8, p. 843-857, 2001. http://dx.doi. org/10.1108/03090560110396269

MUNDIM, A. P. F. et al. Aplicando o cenário de desenvolvimento de produtos em um case prático de capacitação profissional. Gestão \& Produção, v. 9, n. 1, p. $1-16,2002$

NEVES, S. M. et al. Contribuição da gestão do conhecimento para a tomada de decisão e gerenciamento de riscos em projetos. In: SIMPÓSIO DE ENGENHARIA DE PRODUÇÃO - SIMPEP, 16., 2009, Bauru. Anais... Bauru, 2009. p. 1-12.

PAHL, G. et al. Projeto na engenharia: fundamentos do desenvolvimento eficaz de produtos, métodos e aplicações. São Paulo: Edgard Blücher, 2005.

PIRES, S. R. 1.; SACOMANO NETO, M. New configurations in supply chains: the case of a condominium in Brazil's automotive industry. Supply Chain Management: an International Journal, v. 13, n. 4, p. 328-334, 2008. http://dx.doi.org/10.1108/13598540810882215

POPADIUK, S. et al. Ambiente informacional e desempenho competitivo na indústria de autopeças para veículos. Produção, v. 15, n. 3, p. 390-403, 2005. 
QUINTELLA, H. L. M. M.; ROCHA; H. M.; ALVES, M. F. Projeto de veículos automotores: fatores críticos de sucesso no lançamento. Produção, v. 15, n. 3, p. 334-346, 2005. http://dx.doi.org/10.1590/S0103-65132005000300005

QUINTELLA, H. L. M. M.; ROCHA; H. M. Avaliação da maturidade do processo de desenvolvimento de veículos automotivos. Gestão \& Produção, v. 13, n. 2, p. 297-310, 2006. http://dx.doi.org/10.1590/S0104530X2006000200011

RACHID, A.; BRESCIANI FILHO, E.; GITAHY, L. Relações entre grandes e pequenas empresas de autopeças e a difusão de práticas de gestão da produção. Gestão \& Produção, v. 8, n. 3, p. 319-333, 2001. http://dx.doi.org/10.1590/ S0104-530X2001000300008

ROMANO, L. N. Modelo de referência para o desenvolvimento de máquinas agrícolas. 2003. Tese (Doutorado em Engenharia Mecânica)-Universidade Federal de Santa Catarina, Florianopolis, 2003.

ROSA, E. P. S.; SELLITO, M. A.; MENDES, L. W. Avaliação multicriterial de desempenho e separação em aglomerados de fornecedores críticos de uma manufatura OKP. Produção, v. 16, n. 3, p. 413-428, 2006.

ROSENTHAL, S. R. Effective product design and development. How to cut lead time and increase customer satisfaction. Illinois: Business One Irwin, 1992.

ROZENFELD, H. et al. Gestão de desenvolvimento de produtos: Uma referência para a melhoria do processo. São Paulo: Saraiva, 2006.

SAATY, T. L. Método de análise hierárquica. São Paulo: McGraw-Hill, 1991.

SAATY, T. L. Decision-making with the AHP: why is the principal eigenvector necessary. European Journal of Operational Research, v. 145, n. 1, p. 85-91, 2003. http:// dx.doi.org/10.1016/S0377-2217(02)00227-8

SAATY, T. L. Decision making with the analytic hierarchy process. International Journal Services Sciences, v. 1, n. 1, p. 83-98, 2008. http://dx.doi.org/10.1504/ 1JSSCI.2008.017590

SAATY, T.L.; SHANG, J. S. Group decision-making: Head-count versus intensity of preference. Socio-Economic Planning Sciences, v. 41, n. 1, p. 22-37, 2007. http://dx.doi. org/10.1016/j.seps.2005.10.001

SALERNO, M. S. et al. A nova configuração da cadeia automotiva brasileira. Poli/USP e BNDES, 2002. Relatório de Pesquisa DEP.
SALOMON, V. P.; MONTEVECHI, J. A. B. Método de Análise em Redes: o Sucessor do Método de Análise Hierárquica? ln: ENCONTRO NACIONAL DE ENGENHARIA DE PRODUÇÃO, 17., 1997, Gramado. Anais... Gramado, 1997.

SAlOMON, V. A. P.; MONTEVECHI, J. A. B.; PAMPLONA, E. O. Justificativas para aplicação do método de análise hierárquica. In: CONGRESSO NACIONAL DE ENGENHARIA DE PRODUÇÃO, 19., 1999, Rio de Janeiro. Anais... Rio de Janeiro, 1999.

SALOMON, V. A. P. Desempenho da modelagem do auxílio à decisão por múltiplos critérios na análise do planejamento e controle da produção. 2004. Tese (Doutorado em Engenharia)-Escola Politécnica, Universidade de São Paulo, São Paulo, 2004.

SEIDEL, M.; LOCH, C. H.; CHAHIL, S. Quo vadis, automotive industry? A vision of possible industry transformation. European Management Journal, v. 23, n. 4, p. 439-449, 2005. http://dx.doi.org/10.1016/j. emj.2005.06.005

SILVEIRA, M. A. P.; DINIZ, E. H. Relação entre mudança organizacional e implantação de sistemas de informações: um estudo no setor de autopeças. Gestão \& Produção, v. 9, n. 3, p. 397-410, 2002. http://dx.doi.org/10.1590/ S0104-530X2002000300012

SINDICATO NACIONAL DA INDÚSTRIA DE COMPONENTES PARA VEÍCULOS AUTOMOTORES - SINDIPEÇAS. Desempenho do setor de autopeças 2008. SINDIPEÇAS, 2008. Disponível em: <http://www. sindipecas.org.br>. Acesso em: abr. 2009.

SUAREZ, T. M.; JUNG, C. F.; CATEN, C. S. T. Adaptação e aplicação de um método de desenvolvimento de produtos em uma microempresa de manufatura de produtos decorativos. Revista $P \& D$ em Engenharia de Produção, v. 7, n. 1, p. 37-63, 2009.

TOLED0, J. C. et al. Práticas de gestão no desenvolvimento de produtos em empresas de autopeças. Produção, v. 18, n. 2, p. 405-422, 2008.

WHEELWRIGHT, S. C.; CLARK, K. B. Revolutionizing product development: Quantum leaps in speed, efficiency and quality. New York: The Free Press, 1992.

WHEELWRIGHT, S. C.; CLARK, K. B. Accelerating the design-build test cycle for effective product development. International Marketing Review, v. 11, n. 1, p. 32-46, 1994. http://dx.doi.org/10.1108/02651339410057509

\title{
Selection of a PDP reference model to an auto parts company by means of multi-criteria techniques
}

\begin{abstract}
The Product Development Process (PDP) is a critical success factor in launching a new product to meet customer requirements. The adoption of a reference model serves as a comprehensive guide to assist organizations in managing and systematization of the PDP, through standardized sequence of steps that are adapted to the type of product that the company develops. The aim of this paper is to select a reference model of the product development process for the auto part sector by establishing an aid to multi-criteria decision known as Analytic Hierarchy Process (AHP). The research method used was modeling, which before six alternatives, three criteria and trial of five experts resulted in the selection of Rozenfeld et al. (2006) reference model as the most suitable model to analyze the company's PDP selected as object of study in future research.
\end{abstract}

Keywords

Product development process management. Reference model. AHP. 